ATMOSPHERIC AND ENVIRONMENTAL RESEARCH, INC.

Progress Report No. 2 (revised)

DOE/ER/61065--2

DE93 001792

on

\title{
Testbed Model and Data Assimilation for ARM
}

A research program funded by

Office of Energy Research

U.S. Department of Energy

through Procurement Instrument

DE-FG02-90ER61065

\author{
Submitted by: \\ Atmospheric and Environmental Research, Inc. \\ For the period \\ 1 March 1991 through 31 August 1992
}

22 September, 1992

DISCLAIMER

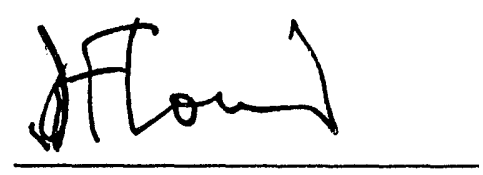

Jean-François Louis

Principal Investigator
This report was prepared as an account of work sponsored by an agency of the United States Government. Neither the United States Government nor any agency thereof, nor any of their employees, makes any warranty, express or implied, or assumes any legal liability or responsibility for the accuracy, completeness, or usefulness of any information, apparatus, product, or process disclosed, or represents that its use would not infringe privately owned rights. Reference herein to any specific commercial product, process, or service by trade name, trademark, manufacturer, or otherwise does not necessarily constitute or imply its endorsement, recommendation, or favoring by the United States Government or any agency thereof. The views and opinions of authors expressed berein do not necessarily state or reflect those of the United States Government or any agency thereof. 


\section{A. Objectives and Work Accomplished}

\section{A.1 Background}

The objectives of this contract are to further develop and test the ALFA (AER Local Forecast and Assimilation) model originally designed at AER for local weather prediction and apply it to three distinct but related purposes in connection with the Atmospheric Radiation Measurement (ARM) program: (a) to provide a testbed that simulates a global climate model in order to facilitate the development and testing of new cloud parametrizations and radiation models; (b) to assimilate the ARM data continuously at the scale of a climate model, using the adjoint method, thus providing the initial conditions and verification data for testing parametrizations; (c) to study the sensitivity of a radiation scheme to cloud parameters, again using the adjoint method, thus demonstrating the usefulness of the testbed model.

The data assimilation will use a variational technique that minimizes the difference between the model results and the observation during the analysis period. The adjoint model is used to compute the gradient of a measure of the model errors with respect to nudging terms that are added to the equations to force the model output closer to the data.

The radiation scheme that will be included in the basic ALFA model makes use of a generalized two-stream approximation, and is designed for vertically inhomogeneous, multiple-scattering atmospheres. The sensitivity of this model to the definition of cloud parameters will be studied. The adjoint technique will also be used to compute the sensitivities.

This project is designed to provide the Science Team members with the appropriate tools and modeling environment for proper testing and tuning of new radiation models and cloud parametrization schemes.

\section{A.2 Progress During the Reporting Period}

The plan for the first year of the project was to incorporate the radiation code of Toon, et al. into the ALFA model, to write its adjoint and to modify the model to be able to use it in data assimilation. This work has been done. As part of this work, code modularity was enforced to enable easy addition or replacement of parts of the code.

During the second year of the contract we have spent a fair amount of time checking the accuracy of the radiation code by trying to simulate the evolution of the atmospheric 
temperature measured during the Wangara campaign. Initially, we found that the code produced too much cooling of the ground surface and atmospheric boundary layer during the night. When we tried to reproduce the radiative fluxes computed by detailed line-by-line radiation codes for standard profiles in clear air, we found that the Toon code underestimated the downward infrared flux. This problem was eventually traced, by one of T. Ackerman's students, to an error in the spectral data for the water vapor continuum. After correcting this error, the code now reproduces well the line-by-line computations and simulates the Wangara data reasonably well.

Testing the correctness of the adjoint code has proven to be a more difficult work then expected. Writing the adjoint involves first writing the tangent linear model (TLM), then transposing it and reversing the operations to obtain the adjoint. The TLM has the same structure as the nonlinear model, and the main source of errors for the TLM is in writing the derivatives of the model equations. To check it, one compares the gradient computed by the TLM with the gradient computed by finite difference, using the nonlinear model. For a short integration, the two gradients should be very similar, although not perfectly equal.

To check the adjoint, one can compare the gradient obtained as a solution of the adjoint with the gradient computed by integrating the TLM with small initial perturbation in each control variable as initial conditions ( $n$ integrations of the TLM are required to compute the whole gradient if we have $\mathrm{n}$ control variables). In this case the gradients should be identical, to within the precision of the computer. Unfortunately, when the gradients are not identical, this test gives no indication about where in the code the error(s) might be. One must then divide the code in smaller portions, compute an appropriate cost function at the point where the code is divided, and repeat the procedure until the erroneous code is isolated. This is a procedure that is very time consuming. It took about one month to find an error of logic in the portion of the code that defines the wavenumber integration intervals in the routine that calls the radiation. Although this time was basically wasted, it taught us a great deal about writing and testing adjoint codes!

We have started defining the structure of the data assimilation system and writing the code for a "supervisor" that will control all the necessary operations: ingesting and checking the data, running the model, storing the results, comparing them with the observations to compute the objective function, running the adjoint model, performing the minimization of the objective function, and handling the user interface.

We have also started writing the adjoint for the moist processes in our model. We wrote the adjoint of the Kuo convection scheme and the stratiform precipitation scheme. Both of 
these phenomena are characterized by threshold processes, with different behaviors when some model variables reach some critical values. Stratiform precipitation starts when the specific humidity reaches saturation, and convection requires positive moisture convergence and conditional instability. This means that the model equations are only piecewise continuous, and that their derivatives with respect to the model variables are not defined at these threshold values. This may impact the parameter optimization process by creating local minima in the cost function adversely affecting its minimization.

We have chosen two different strategies for stratiform precipitation and convection. For stratiform precipitation, it is relatively easy to write the equation as a continuous process, by replacing the instantaneous adjustment by an algorithm that removes a fraction of the existing moisture at each time step, the fraction removed being a strong function of relative humidity.

We write the precipitation tendency as:

$$
\left(\frac{\partial q}{\partial t}\right)_{\text {precip }}= \begin{cases}\frac{1}{\tau}\left[-\left(q-q_{s}\right)-q_{s}(1-c) \mathrm{e}^{\left.-\frac{q-c q_{s}}{(1-c) q_{s}}\right],}\right. & q>c q_{s} \\ 0, & q \leq c q_{s}\end{cases}
$$

where $q$ is the specific humidity, $q_{s}$ its saturation value, $\tau$ an adjustable time constant, and $c$ is an adjustable constant somewhat similar to a critical relative humidity. Figure 1 shows how much moisture would be removed in one time step, in terms of the relative humidity, if the time constant $\tau$ were equal to the time step and $c=1$. It is not very different from simply removing the supersaturated fraction, but the two adjustable constant give us more flexibility. The derivatives of this expression with respect to $q, q_{s}$ and $c$ are all equal to zero for $q=c q_{s}$. Therefore the derivatives are continuous, even though we have different expressions in the two regimes.

It is more difficult to write a moist convection scheme as a continuous process. Furthermore, it can be argued that the life time of convective cells is of the same order as the model time step (about 15 minutes). Therefore, from the model's point of view, convection really is a discontinuous phenomenon. We decided to keep the Kuo scheme as it is, and write its adjoint. Derivatives can be written in both convective and non-convective regimes. It is only at the transition that the derivatives are undefined. We will carefully test whether this actually creates problems of convergence for the minimization. 


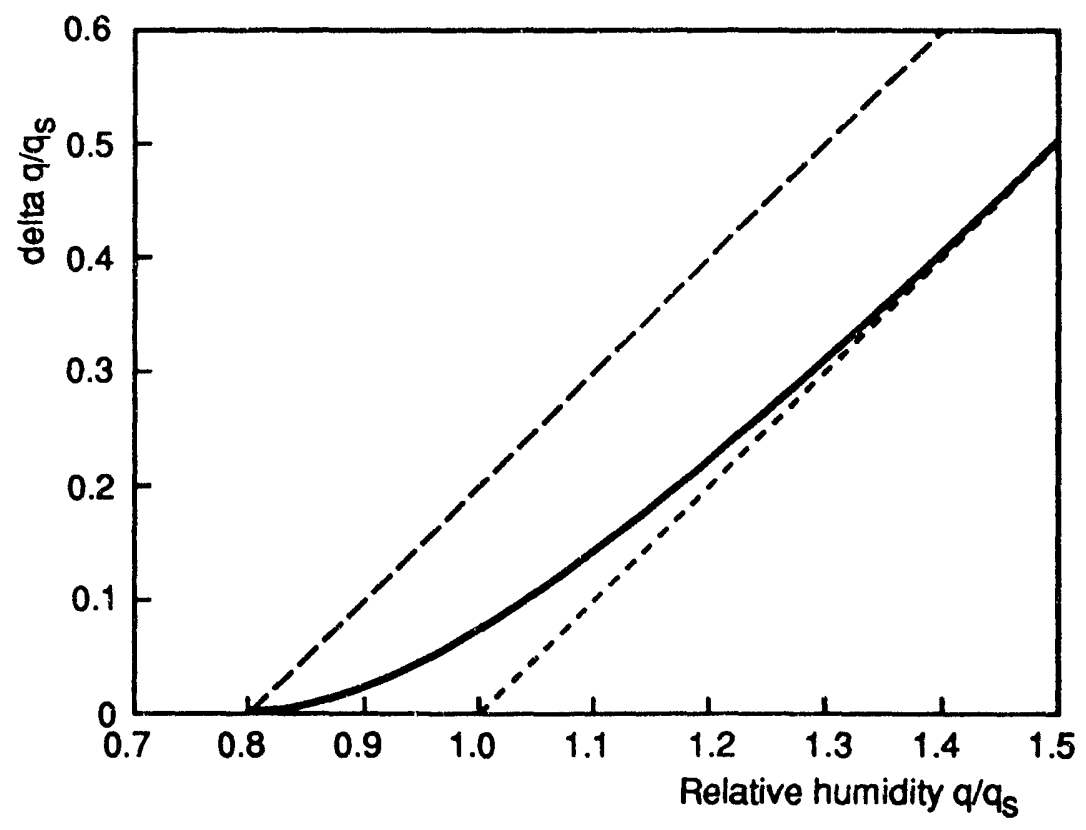

Figure 1: Change of relative humidity in one time step. Solid line: amount removed according to Eq. (16) if $t=\mathrm{dt}$ and $\mathrm{c}=.8$. Dashed lines: amount removed by standard method with critical relative humidity of 1 (short dashes) or 0.8 (long dashes).

Under another contract (NSF SBIR), we have started accumulating all the standard meteorological data, as well as the NMC analyses and forecasts for North America. These data will be used to test our system prior to the start of the CART site operations.

\section{B. Equipment and Property Acquired}

None

\section{Travel}

Travel since the 1991 progress report :

- June 18-21, 1991, La Jolla, CA, : JASON meeting on remotely piloted vehicles and small satellites. (J-F. Louis)

- September 16-20, 1991, Livermore, CA: meeting of working group on measurement strategies. (J-F. Louis)

- October 26-30, 1991, Denver, CO: annual ARM science team meeting. (J-F. Louis)

- February 18-20, 1992, Santa Barbara, CA: invited by C. Gautier to give a minicourse on adjoint methods at UCSB. (J-F. Louis) 
- April 6-10, 1992, Edinburgh, UK: annual meeting of the European Geophysical Society. (J-F. Louis)

- August 23-28, 1992, Monterey, CA: workshop on the use of adjoint methods in meteorology, organized by Ron Errico (NCAR), Philippe Courtier (ECMWF) and Jean-François Louis (AER) (J-F. Louis and Marina Živkovic)

\section{Plans for the Rest of the Period}

The remainder of year 2 will be devoted mainly to setting up and testing the data assimilation system. These tests will be done with both simulated and real data. We will also improve the user interface of the model.

\section{E. Publications and Presentations}

Jean-François Louis gave a mini-course on adjoint methods in meteorology at the university of California, Santa Barbara.

A paper, entitled "A method for implementing adjoint calculations in the discrete case", by Ross N. Hoffman, Jean-François Louis and Thomas Nehrkorn was published as ECMWF RD Technical Memorandum No. 184. (Available from ECMWF, Reading, Berks., UK) 

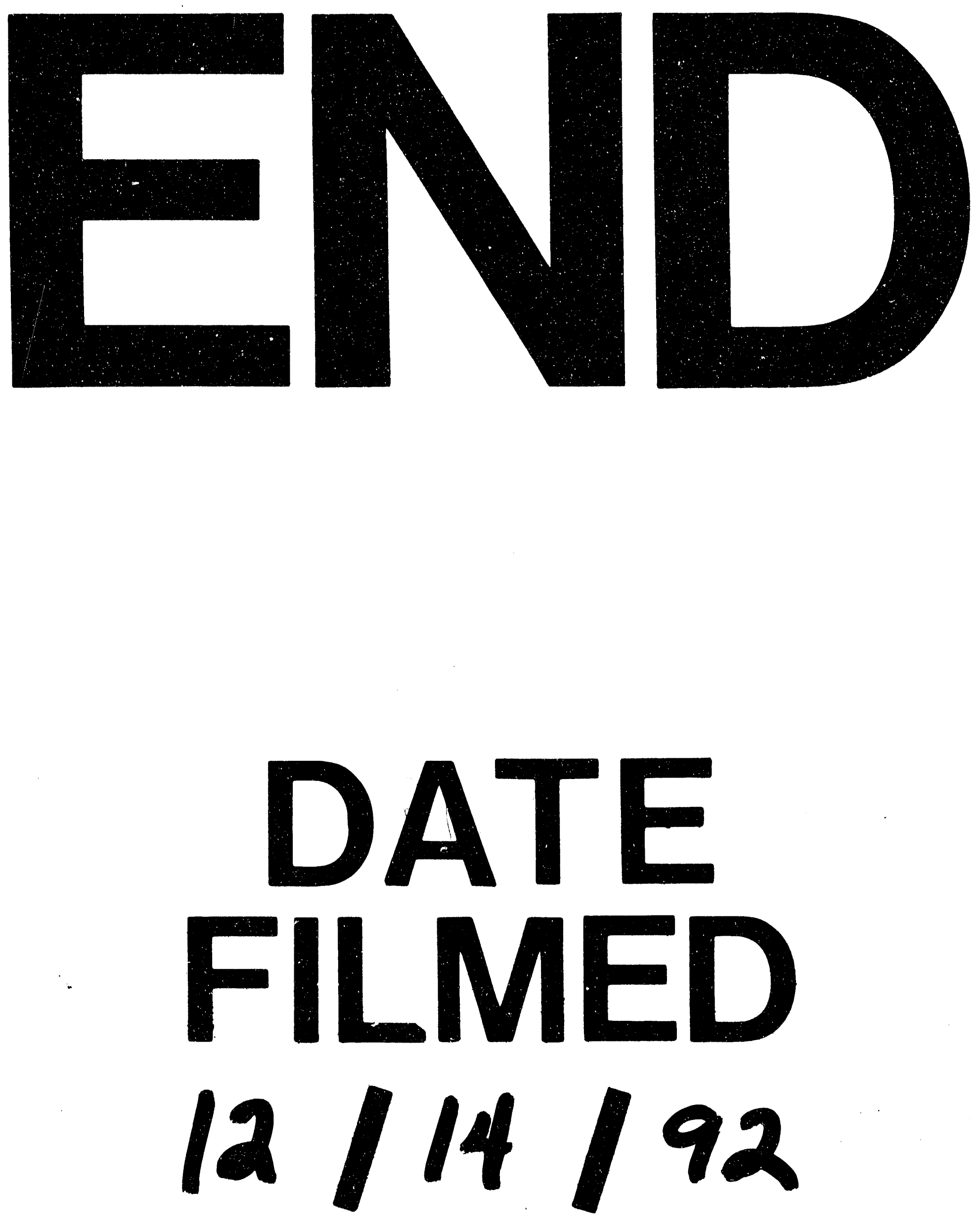
